\title{
CYP1B1 Activates Wnt/ $\beta$-Catenin Signaling through Suppression of Herc5-Mediated ISGylation for Protein Degradation on $\beta$-Catenin in HeLa Cells
}

\author{
Young-Shin Park ${ }^{\dagger}$, Yeo-Jung Kwon ${ }^{\dagger}$ and Young-Jin Chun \\ College of Pharmacy, Chung-Ang University, Seoul, Korea
}

\begin{abstract}
Cytochrome P450 1B1 (CYP1B1) acts as a hydroxylase for estrogen and activates potential carcinogens. Moreover, its expression in tumor tissues is much higher than that in normal tissues. Despite this association between CYP1B1 and cancer, the detailed molecular mechanism of CYP1B1 on cancer progression in HeLa cells remains unknown. Previous reports indicated that the mRNA expression level of Herc5, an E3 ligase for ISGylation, is promoted by CYP1B1 suppression using specific small interfering RNA, and that ISGylation may be involved in ubiquitination related to $\beta$-catenin degradation. With this background, we investigated the relationships among CYP1B1, Herc5, and $\beta$-catenin. RT-PCR and western blot analyses showed that CYP1B1 overexpression induced and CYP1B1 inhibition reduced, respectively, the expression of Wnt $/ \beta$-catenin signaling target genes including $\beta$-catenin and cyclin D1. Moreover, HeLa cells were treated with the CYP1B1 inducer 7,12-dimethylbenz[ $\alpha]$ anthracene (DMBA) or the CYP1B1 specific inhibitor, tetramethoxystilbene (TMS) and consequently DMBA increased and TMS decreased $\beta$-catenin and cyclin D1 expression, respectively. To determine the correlation between CYP1B1 expression and ISGylation, the expression of ISG15, a ubiquitin-like protein, was detected following CYP1B1 regulation, which revealed that CYP1B1 may inhibit ISGylation through suppression of ISG15 expression. In addition, the mRNA and protein expression levels of Herc 5 were strongly suppressed by CYP1B1. Finally, an immunoprecipitation assay revealed a direct physical interaction between Herc5 and $\beta$-catenin in HeLa cells. In conclusion, these data suggest that CYP1B1 may activate Wnt/ $\beta$-catenin signaling through stabilization of $\beta$-catenin protein from Herc5-mediated ISGylation for proteosomal degradation.
\end{abstract}

Key words: CYP1B1, $\beta$-Catenin, Herc-5, ISGylation

\section{INTRODUCTION}

Cytochrome P450s are members of a superfamily group composed of monooxygenases (1). Specifically, P450 enzymes catalyze the metabolic processes of various types of substances, including eicosanoids, steroids, fatty acids, xenobiotics, and chemical carcinogens $(2,3)$.

Correspondence to: Young-Jin Chun, College of Pharmacy, ChungAng University, 84 Heukseok-ro, Dongjak-gu, Seoul 06974, Korea E-mail: yjchun@cau.ac.kr

${ }^{\dagger}$ These authors contributed equally to this study.

This is an Open-Access article distributed under the terms of the Creative Commons Attribution Non-Commercial License (http:// creativecommons.org/licenses/by-nc/3.0) which permits unrestricted non-commercial use, distribution, and reproduction in any medium, provided the original work is properly cited.
Cytochrome P450 1B1 (CYP1B1), a monooxygenase belonging to the cytochrome P450 1 superfamily, acts as a hydroxylase in estrogen metabolism (4). The major function of CYP1B1 in humans is to switch $17 \beta$-estradiol into 4-hydroxyestradiol (5). CYP1B1 is known as an oncogenic protein based on its ability to activate several carcinogens derived from environmental sources (6). Furthermore, the tissue-specific expression levels of CYP1B1 have been reported to be much higher in tumors than in normal tissues (7). The potential association between CYP1B1 and cancer has been widely suggested for decades; however, the detailed mechanistic explanation of CYP1B1 function in tumor progression is still not fully established.

Wnt/ $\beta$-catenin signaling has been identified to play a critical role in tumorigenesis and biological processes associated with cell development (8). In addition, Wnt/ $\beta$-catenin signals are well-known stimuli for the induction of cell pro- 
liferation and controlling the differentiation of post-mitotic cells (9). The canonical Wnt/ $\beta$-catenin pathway is initiated by Wnt ligand binding to its transmembrane receptors, which leads to multiple signal transductions in the cytosol to induce the nuclear translocation of $\beta$-catenin and ultimately regulate target gene expression (10). During the signal transduction processes of the $\mathrm{Wnt} / \beta$-catenin pathway following Wnt ligand binding to Frizzled receptor and LRP6, the receptors are phosphorylated by GSK3 $\beta$ and casein kinase $\gamma$ in the cytoplasmic region, which initiates the recruitment of Dishevelled 1-3 and Axin. Consequently, $\beta$-catenin avoids phosphorylation and its degradation is thus undermined. Intact $\beta$-catenin is able to translocate into the nucleus and modulate target gene expression via $\mathrm{T}$ cell-specific transcription factor/lymphoid enhancer-binding factor sites (10). Thus, cytoplasmic $\beta$-catenin acts as a critical regulator in the Wnt signaling pathway, and there have been several reports showing that $\beta$-catenin is linked to diverse types of human diseases, including cancer, neuro-degradation, and osteoporosis (11).

Recently, it has been revealed that interferon-stimulated gene 15 (ISG15) conjugation is involved in the degradation of $\beta$-catenin (12). ISG15, a ubiquitin-like protein (Ubl), has two domains and a structure similar to ubiquitin, and the conserved C-terminal motif of ISG15 is known to be critical for conjugation on target proteins (13). Like ubiquitination, the ISGylation system is proceeded by the action of E1 (Ube1L), E2 (UbcH8), and E3 ligase, and this protein modification is known to be stimulated by interferons (IFNs) (14). More than 100 proteins have been identified as potential targets of ISG15 conjugation (15). Moreover, several recent studies reported that newly synthesized proteins identified in viralinfected cells may be the main targets of ISG15 $(16,17)$.

Herc5, a member of the HERC protein family, is distinguished from other members in the group by possession of a domain homologous to the E6-AP carboxyl terminus (HECT) domain and RCC1-like domains (RLD) (18). The possibility that HERC proteins act as E3 ligases in biological protein degradations has been recently suggested (19). In particular, it has been revealed that Herc5 functions as an E3 ligase for ISG15 to induce ISGylation for target protein degradation, and this process is highly activated by type I IFNs (20). Herc5 is reported to show high-level expression in the fetal brain and testis, but is expressed at low levels in most other tissues (21). A recent study showed that the mRNA expression level of Herc5 was significantly promoted by $C Y P 1 B 1$ knockdown using specific small interfering RNA (siRNA) (4). In this regard, further investigation is needed to obtain a detailed understanding of the relationship between CYP1B1 and Herc5.

In the present study, we explored the role of CYP1B1 on the $\mathrm{Wnt} / \beta$-catenin signaling pathway in the human cervical cancer cell line HeLa. Among the target genes of $\beta$-catenin, cyclin D1 was chosen to be detected for validation of activated status of $\mathrm{Wnt} / \beta$-catenin signaling due to its correlation with cell proliferation-based carcinogenesis. Furthermore, the effect of CYP1B1 on Herc5 and ISG15 expression was evaluated. Since Herc5 has the ability to act as an E3 ligase for ISGylation, we further investigated whether Herc 5 is involved in the action of CYP1B1 in the $\mathrm{Wnt} / \beta$-catenin signaling pathway.

\section{MATERIALS AND METHODS}

Materials. Antibodies for CYP1B1, $\beta$-catenin, cyclin D1, Herc5, ISG15, and $\beta$-actin, and the horseradish peroxidase (HRP)-conjugated mouse anti-goat IgG secondary antibody and normal rabbit IgG were obtained from Santa Cruz Biotechnology, Inc (Santa Cruz, CA, USA). HRPconjugated goat anti-rabbit or mouse IgG secondary antibodies were purchased from Abclon Biotechnology (Seoul, Korea). Enhanced chemiluminescence (ECL) reagent was obtained from Thermo Scientific (Rockford, IL, USA). 7,12Dimethylbenz $[\alpha]$ anthracene (DMBA) was purchased from Sigma-Aldrich (St. Louis, MO, USA). 2,2',4,6'-Tetramethoxystilbene (TMS) was generously provided by Dr. Sanghee Kim (Seoul National University, Seoul, Korea). The specific siRNA for CYP1B1 was obtained from Qiagen (Hilden, Germany) and scrambled siRNA for control was purchased from Bioneer (Daejeon, Korea). M-MLV reverse transcriptase and the RNase inhibitor were purchased from Promega (Madison, WI, USA). Ex Taq polymerase and SYBR premix Ex Taq were obtained from TaKaRa Bio (Shiga, Japan). Other chemicals and reagents were of the highest quality commercially available.

Cell culture. The human cervical cancer cell line HeLa was purchased from the Korean Cell Line Bank (KCLB). Cells were cultured in minimal essential medium (MEM) with Earle's Balanced Salts medium supplemented with $10 \%$ heat-inactivated fetal bovine serum, $100 \mathrm{U} / \mathrm{mL}$ penicillin, and $100 \mu \mathrm{g} / \mathrm{mL}$ streptomycin in a constant humidified atmosphere of $5 \% \mathrm{CO}_{2}$ at $37^{\circ} \mathrm{C}$. After treatment with the chemicals, the cells were harvested by scraping and collected by centrifugation at $1,000 \times g$ for $5 \mathrm{~min}$ at $4^{\circ} \mathrm{C}$, and the pellets were stored at $-20^{\circ} \mathrm{C}$.

Transient transfection of plasmid DNA or siRNA. Cells were collected at a density of approximately $1 \times 10^{6}$ cells per $1.5-\mathrm{mL}$ tube. Transfection was carried out using the $\mathrm{Neon}^{\mathrm{TM}}$ transfection system (Invitrogen, Carlsbad, CA, USA), and $1 \times 10^{6}$ cells were transfected with $5 \mu \mathrm{g}$ of plasmid DNA or $40 \mathrm{nM}$ CYP1B1 specific siRNA or scrambled siRNA as a control. Neon ${ }^{\text {TM }}$ Transfection System was used in accordance with the manufacturer's protocol for HeLa cells with two pulses of $1005 \mathrm{~V}$ for $35 \mathrm{~ms}$. For CYP1B1 overexpression, CYP1B1 cDNA was subcloned into the pcDNA3.1/zeo plasmid vector. The CYPIB1 specific siRNA target sequence was as follows: 5'-CAGCATGATG- 
CGCAACTTCTT-3'. Following electroporation, the cells were seeded in 100-mm dishes and cultured with antibioticfree MEM. After $6 \mathrm{hr}$, the medium containing antibiotics was added and the cells were incubated further for $48 \mathrm{hr}$ at $37^{\circ} \mathrm{C}$ in $5 \% \mathrm{CO}_{2}$.

Reverse transcription-polymerase chain reaction (RTPCR). After harvesting of cells, total RNA was isolated using Ribospin ${ }^{\mathrm{TM}}$ (GeneALL, Seoul, Korea). RT for total RNA (500 ng) was performed at $37^{\circ} \mathrm{C}$ for $1 \mathrm{hr}$ in a $25-\mu \mathrm{L}$ reaction mixture containing $5 \mathrm{X}$ RT buffer, OligodT, dNTPs, RNase inhibitor, and Moloney murine leukemia virus (MMLV) reverse transcriptase. Subsequently, $0.8 \mu \mathrm{L}$ of the cDNA was amplified with 10 pmol of specific primer pairs, dNTPs, 10X Ex Taq buffer, and Ex Taq polymerase in a final volume of $20 \mu \mathrm{L}$. PCR cycles were performed as follows: $95^{\circ} \mathrm{C}$ for $5 \mathrm{~min}$, followed by 35 cycles of denaturation at $95^{\circ} \mathrm{C}$ for $10 \mathrm{~s}$, annealing at $60^{\circ} \mathrm{C}$ for $15 \mathrm{~s}$, and elongation at $72^{\circ} \mathrm{C}$ for $15 \mathrm{~s}$. The number of cycles for gene amplification was optimized in preliminary experiments to confirm that the PCR amplification did not reach a plateau. PCR products were subjected to $2 \%$ agarose gel electrophoresis and detected by ChemiDoc XRS (Bio-Rad, Hercules, CA, USA). The sequences of primers used in this study are described in Table 1.

Quantitative PCR (qPCR). qPCR was performed with CFX Connect ${ }^{\mathrm{TM}}$ Real-Time PCR Detection System (Bio$\mathrm{Rad})$. The $\mathrm{qPCR}$ mixture containing $10 \mu \mathrm{L}$ of the $2 \mathrm{X}$ SYBR green Premix Ex Taq, 10 pmol of specific primer pairs, and $2 \mu \mathrm{L}$ of diluted cDNA (1:10 dilution) was prepared in a final volume of $20 \mu \mathrm{L}$. PCR cycles were carried out as described above for RT-PCR. Relative changes in gene expression were analyzed using the comparative cycle threshold method. GAPDH was used as an internal control.

Western blot analysis. After harvesting, the cells were lysed in $50 \mathrm{mM}$ Tris- $\mathrm{HCl}$ (pH 8.0) containing $150 \mathrm{mM}$ $\mathrm{NaCl}, 1 \%$ nonidet P-40, protease inhibitor cocktail, $1 \mathrm{mM}$ phenylmethane sulfonyl fluoride, $25 \mathrm{mM}$ sodium fluoride,

Table 1. Sequences of PCR primers

\begin{tabular}{|c|c|}
\hline Name & Sequence \\
\hline \multirow{2}{*}{ CYP1B1 } & Forward: 5'-TGTCCTGGCCTTCCTTTATGA-3' \\
\hline & Reverse: 5'-TCCTTGTTGATGAGGCCATC-3' \\
\hline \multirow{2}{*}{$\beta$-Catenin } & :'-ATGTCCAGCGTTTGGCTGAA-3' \\
\hline & -TGGTCCTCGTCATTTAGCAGTT-3' \\
\hline \multirow{2}{*}{ Cyclin D1 } & CCTGAGC \\
\hline & 'TCTGCTCCTGGCAGGCC-3' \\
\hline \multirow{2}{*}{ Herc5 } & Forward: 5'-GGGATGAAAGTGCTGAGGAG-3' \\
\hline & Reverse: 5'-CATTTTCTGAAGCGTCCACA-3' \\
\hline \multirow{2}{*}{ GAPDH } & Forward: 5'-TGAACGGGAAGCTCACTG-3' \\
\hline & Reverse: 5'-TCCACCACCCTGTTGCTGTA-3' \\
\hline
\end{tabular}

and $1 \mathrm{mM}$ sodium orthovanadate. Protein concentrations were determined using BCA Protein Assay Kit (Pierce, Rockford, IL, USA). Subsequently, cellular extracts (45 60 $\mu \mathrm{g})$ were separated with sodium dodecyl sulfate-polyacrylamide gel electrophoresis and transferred onto a polyvinylidene fluoride membrane. Non-specific binding was blocked with $5 \%$ skim milk in Tris-buffered saline with Tween 20 for $30 \mathrm{~min}$ at room temperature. The membranes were then incubated with the primary antibody $(1: 1,000)$ overnight at $4^{\circ} \mathrm{C}$ and continually with the secondary antibody $(1: 5,000)$ at room temperature for $30 \mathrm{~min}$. After incubation, the membranes were washed three times for 10 min each. Proteins were visualized using ECL reagent, and the band intensity was measured using a Chemidoc XRS densitometer system and quantified by Quantity One software (Bio-Rad).

Immunoprecipitation (IP). The IP assay was carried out using the Catch and Release ${ }^{\circledR}$ v2.0 kit from Upstate Biotechnology (Lake Placid, NY, USA) according to the manufacturer's protocol. Naive HeLa cell lysates $(500 \mu \mathrm{g})$ were immunoprecipitated with anti-goat Herc5 antibody, using $2 \mu \mathrm{g}$ of a specific Herc5 antibody. Immunoprecipitates were blotted with anti-rabbit $\beta$-catenin antibody. The input represented $50 \mu \mathrm{g}$ of cell lysates. Proteins were detected by western blot analysis as described above.

Statistical analyses. Statistical analyses were performed using one-way analysis of variance, followed by Dunnett's multiple comparison $t$-test when appropriate using GraphPad Prism software (GraphPad Software Inc., San Diego, CA, USA). The differences were considered statistically significant at $p<0.05$.

\section{RESULTS}

CYP1B1 promotes the expression $\beta$-catenin and cyclin D1 in human cervical cancer cells. CYP1B1 is excessively expressed in many types of human tumors and is widely reported to activate potential carcinogens $(4,7)$. The major function of CYP1B1 is the hydroxylation of estradiol. The Wnt/ $\beta$-catenin signaling pathway is one of the most well-known biological signal transductions, playing a key role in cell proliferation and differentiation (9). To elucidate the relationship between CYP1B1 and Wnt/ $\beta$-catenin signaling, HeLa cells, a human cervical cancer cell line, were transfected with a CYP1B1 overexpression vector, and the mRNA expression levels of CYPIBI were measured to validate the transfection efficiency (Fig. 1A, 1B). Subsequently, the protein expression levels of the key regulator and target gene of $\mathrm{Wnt} / \beta$-catenin signaling, $\beta$-catenin and cyclin D1, respectively, were detected by western blot analyses, which showed that CYP1B1 significantly up-regulated both factors (Fig. 1C). To further assess the effect of 
(A)

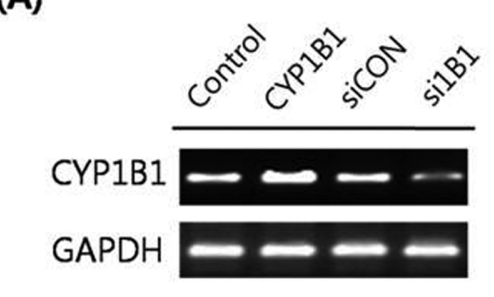

(B)

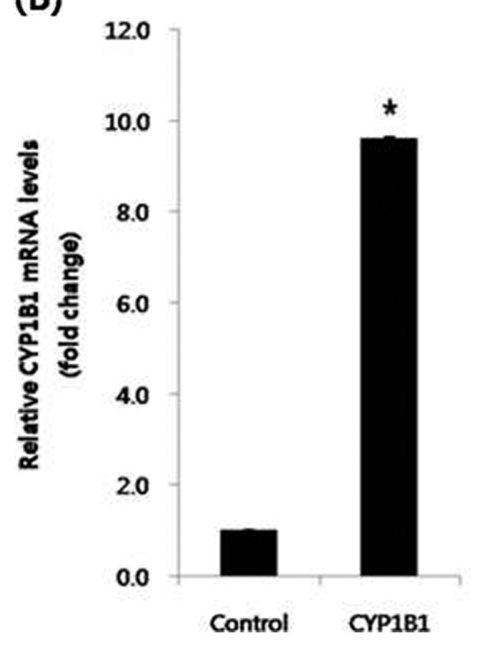

(C)
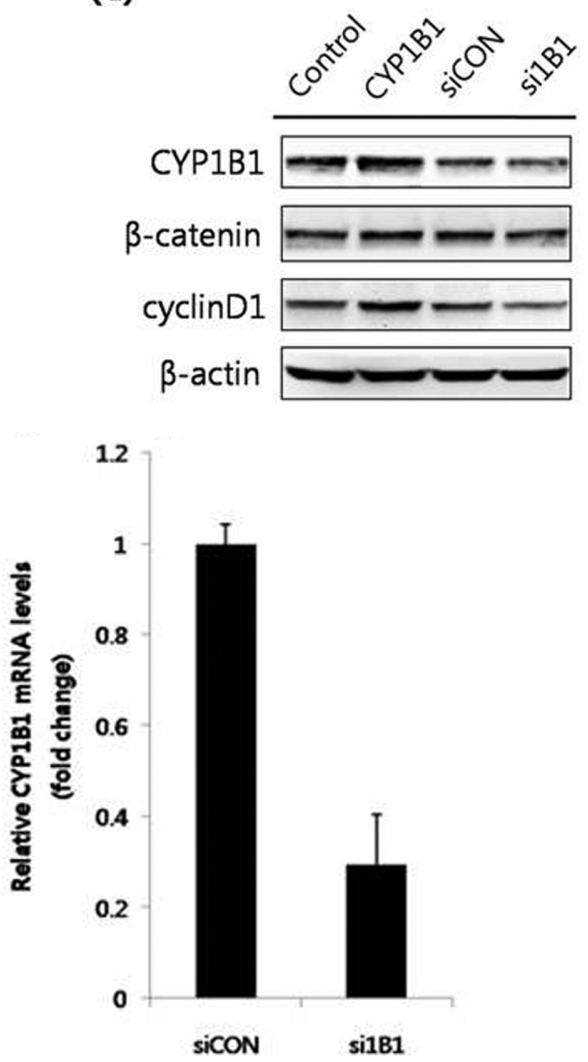

Fig. 1. CYP1B1 promotes the protein expression levels of $\beta$-catenin and cyclin D1 in HeLa cells. (A) RT-PCR analyses. HeLa cells were transfected with pcDNA3.1(zeo)-CYP1B1 $(5 \mu \mathrm{g})$ or CYP1B1 specific siRNA $(40 \mathrm{nM})$ for $48 \mathrm{hr}$. After RNA isolation, gene amplification of CYP1B1 was conducted. GAPDH mRNA was determined as a control. (B) Quantitative PCR analyses. Total RNA was isolated and qPCR for CYP1B1 was performed in triplicate. Data were normalized to the GAPDH value. The data represent mean \pm SD. ${ }^{*} p \leq 0.05$. (C) Western blot analyses. The cells were transfected with pcDNA 3.1(zeo)-CYP1B1 (5 $\mu \mathrm{g}$ ) or CYP1B1 specific siRNA (40 nM). After stabilization for $48 \mathrm{hr}$, total protein was isolated from cell lysates, and the protein expression levels of CYP1B1, $\beta$-catenin, and cyclin D1 were measured. $\beta$-Actin was used as an internal loading control. Empty vector (pcDNA3.1(zeo))-transfected group was represented as control and CYP1B1-overexpressed group was represented as CYP1B1 in figures. Scrambled siRNA-transfected group was represented as siCON while CYP1B1 specific siRNA-transfected group was represented as si1B1 in figures.

(A)<smiles>Cc1c2ccccc2c(C)c2c1ccc1ccccc12</smiles>

(B)

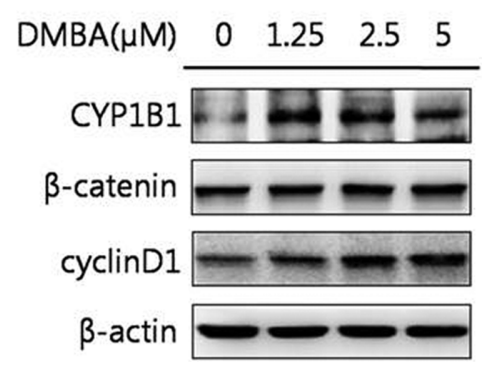

Fig. 2. DMBA, a CYP1B1 inducer, significantly increases the protein expression levels of $\beta$-catenin and cyclin D1. (A) Chemical structure of DMBA. (B) Western blot analyses. HeLa cells were treated with DMBA $(0,1.25,2.5$, or $5 \mu \mathrm{M})$ for $24 \mathrm{hr}$. Total protein was obtained and the protein expression levels of CYP1B1, $\beta$-catenin, and cyclin D1 were detected. $\beta$-Actin was used as an internal loading control.

the increased expression of CYP1B1, the cells were treated with the CYP1B1 inducer DMBA (Fig. 2A). DMBA markedly promoted CYP1B1 expression in a concentration- dependent manner simultaneously with $\beta$-catenin and cyclin D1 expression (Fig. 2B). Suppression of CYP1B1 expression was also achieved by transfection of specific siRNA or 
(A)<smiles>COc1ccc(/C=C/c2c(OC)cccc2OC)c(OC)c1</smiles>

(B)

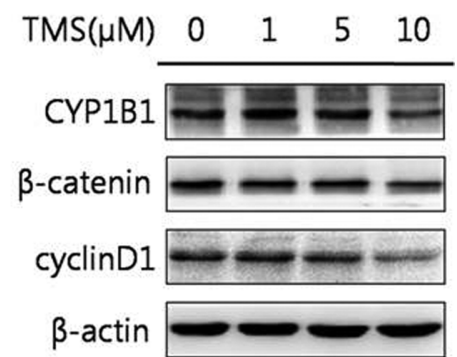

Fig. 3. The selective CYP1B1 inhibitor TMS markedly decreases the expression levels of $\beta$-catenin and cyclin D1. (A) Chemical structure of TMS. (B) Western blot analyses. HeLa cells were treated with TMS $(0,1,5$, or $10 \mu \mathrm{M})$ for $48 \mathrm{hr}$. Cell lysates were isolated. Total protein was isolated and the protein expression levels of CYP1B1, $\beta$-catenin, and cyclin D1 were determined. $\beta$-Actin was used as an internal loading control.

(A)

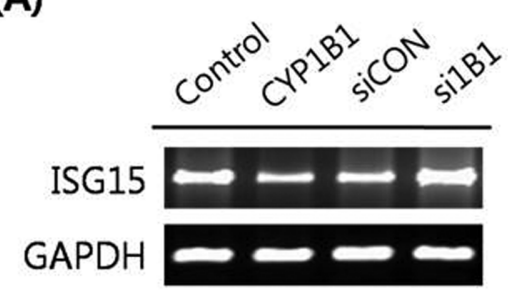

(C)

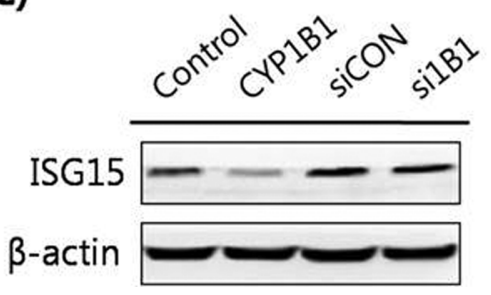

(B)

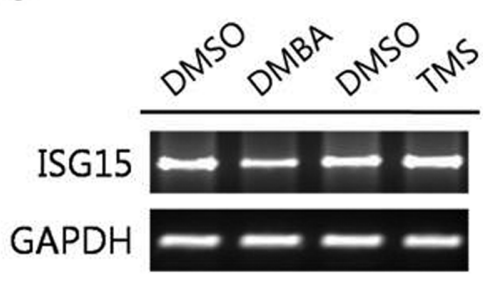

(D)

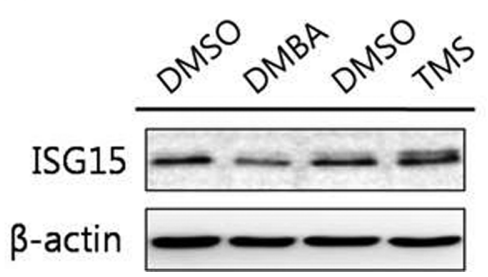

Fig. 4. CYP1B1 strongly suppresses the expression of ISG15 in HeLa cells. (A) RT-PCR analyses. Cells were transfected with pcDNA3.1(zeo)-CYP1B1 $(5 \mu \mathrm{g})$ or CYP1B1 specific siRNA $(40 \mathrm{nM})$ for $48 \mathrm{hr}$. Total RNA was isolated and the ISG15 gene was amplified with specific primers. Expression of GAPDH mRNA was measured as an RNA control. (B) RT-PCR analyses. HeLa cells were treated with DMBA $(5 \mu \mathrm{M})$ for $24 \mathrm{hr}$ or with TMS $(10 \mu \mathrm{M})$ for $48 \mathrm{hr}$. Gene amplification for ISG15 was conducted and GAPDH was amplified as an RNA control. (C) Western blot analyses. HeLa cells were transfected with pcDNA3.1(zeo)-CYP1B1 (5 $\mu \mathrm{g}$ ) or CYP1B1 specific siRNA (40 nM) for $48 \mathrm{hr}$. Cell lysates were obtained and western blot analyses to detect ISG15 were performed. $\beta$-Actin was used as an internal loading control. (D) Western blot analyses. HeLa cells were treated with DMBA $(5 \mu \mathrm{M})$ for $24 \mathrm{hr}$ or with TMS $(10 \mu \mathrm{M})$ for $48 \mathrm{hr}$. Cell lysates were isolated and the protein expression levels of ISG15 were determined. $\beta$-Actin was used as an internal loading control.

treatment with the CYP1B1 specific inhibitor TMS (Fig. $3 \mathrm{~A})$, and the decreased expression level of CYP1B1 was validated by PCR and western blot analyses (Fig. 1, 3B). Similar to the results of the CYP1B1 overexpression experiments, CYP1B1 inhibition massively suppressed the protein expression levels of $\beta$-catenin and cyclin D1 (Fig. 1C, $3 \mathrm{~B})$. These data suggest that CYP1B1 may activate $\mathrm{Wnt} / \beta$ catenin signaling through up-regulation of $\beta$-catenin expression in human cervical cancer cells.

CYP1B1 may be involved in ISGylation through promotion of ISG 15 expression. ISG15, an Ubl protein, has been reported to conjugate onto target proteins for prevent- ing the effects of viral infection (16). Classic $\beta$-catenin protein degradation is orchestrated by the ubiquitinationproteasome pathway using $\beta-\operatorname{TrCP}(21)$. According to Lee et al. (23), the novel mechanism of $\beta$-catenin regulation has been described as ISGylation-dependent proteasomal degradation. As described above, our data showed that the protein expression level of $\beta$-catenin is promoted by CYP1B1. Furthermore, several studies revealed that CYP1B1 takes part in immune responses by acting as a tumor antigen (24), and the innate immunity processes due to viral infection enhance ISGylation (19). Based on our data and these findings, we considered whether CYP1B1 might be involved in ISGylation through ISG15 conjugation. To identify the cor- 
(A)

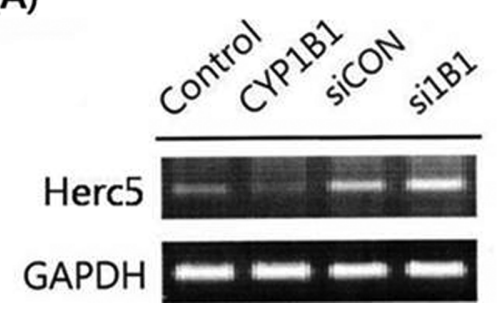

(B)

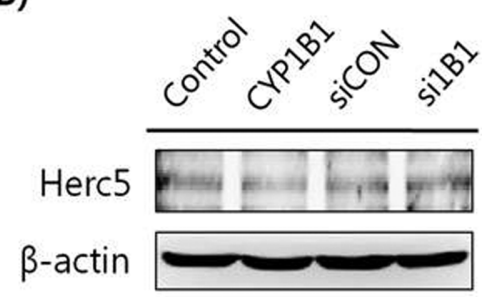

Fig. 5. Herc5 expression is significantly reduced by CYP1B1 in HeLa cells. (A) RT-PCR analyses. Cells were transfected with pcDNA3.1(zeo)-CYP1B1 $(5 \mu \mathrm{g})$ or CYP1B1 specific siRNA $(40 \mathrm{nM})$ for $48 \mathrm{hr}$. Total RNA was isolated and the Herc5 gene was amplified with specific primers. Expression of GAPDH mRNA was measured as an RNA control. (B) Western blot analyses. Cells were transfected with pcDNA3.1(zeo)-CYP1B1 $(5 \mu \mathrm{g})$ or CYP1B1 specific siRNA ( $40 \mathrm{nM})$ for $48 \mathrm{hr}$. Cell lysates were isolated and western blot analyses were performed against Herc5. $\beta$-Actin was used as an internal loading control.

relation between CYP1B1 and the level of ISGylation, the expression levels of ISG15 were determined following CYP1B1 overexpression in HeLa cells. Consequently, significantly suppressed ISG15 expression was observed in CYP1B1-overexpressed cells at both the mRNA and protein levels (Fig. 4A, 4C). Treatment of the CYP1B1 inducer DMBA $(5 \mu \mathrm{M})$ also resulted in massive down-regulation of ISG15 mRNA and protein expression (Fig. 4B, 4D), whereas inhibition of CYP1B1 using specific siRNA and the inhibitor TMS $(10 \mu \mathrm{M})$ markedly induced ISG15 expression (Fig. 4). Taken together, these data suggest that CYP1B1 may enhance the $\beta$-catenin protein level through stabilization of $\beta$-catenin from ISGylation-mediated degradation.

Herc5, an E3 ligase for ISG15, is down-regulated by CYP1B1. Herc5 is known to participate in the ISGylation system as an E3 ligase for ISG15 conjugation (14). According to Saini et al. (4), Herc5 was markedly increased in CYP1B1-knockdown KLE cells, a human endometrium cancer cell line. In addition, we previously identified that CYP1B1 suppressed ISG15 expression levels. To further investigate whether CYP1B1 suppresses ISGylation-executed $\beta$-catenin degradation through regulation of the substrate for conjugation ISG15 simultaneously with the mediator of conjugation Herc5, the effects of CYP1B1 on Herc5 expression were assessed by RT-PCR and western blot analyses in HeLa cells. Similar to the results in KLE cells reported by Saini et al. (4), the Herc5 expression level was increased in CYP1B1-knockdown HeLa cells, while CYP1B1 overexpression strongly decreased Herc5 expression at both the mRNA and protein levels (Fig. 5). Based on these findings, we concluded that CYP1B1 may protect the $\beta$-catenin protein level through inhibition of ISGylation via simultaneously controlling ISG15 and Herc5 expression.

Herc5 may directly interact with and ISGylate onto $\beta$ catenin. To further evaluate whether the hypothesis established in the former section is correct, we investigated the

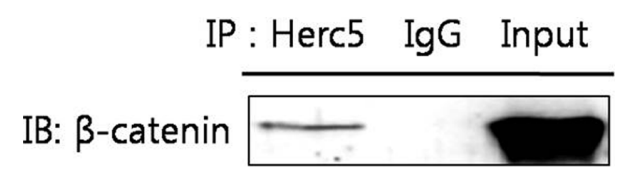

Fig. 6. Herc5 physically interacts with $\beta$-catenin in HeLa cells. The cells were cultured for $48 \mathrm{hr}$ and cell lysates were obtained. Following immunoprecipitation for naive cell lysates $(500 \mu \mathrm{g})$ with anti-goat Herc5 antibody overnight, the immunoprecipitates were subjected to western blot analyses against $\beta$-catenin. The input represented $50 \mu \mathrm{g}$ of cell lysates. IgG was used as a negative control.

possibility that $\beta$-catenin is a target of Herc5. To do so, we conducted an IP assay to detect the existence of a direct interaction between $\beta$-catenin and Herc 5 using western blot analysis with $\beta$-catenin antibody for Herc5-immunoprecipitated cell lysates. As a result, we obtained definitive evidence to prove that $\beta$-catenin and Herc5 physically interact in HeLa cells (Fig. 6). In conclusion, these data suggest that CYP1B1 enhances the $\beta$-catenin protein level through suppression of the ISG15-Herc5-mediated ISGylation of $\beta$-catenin, which ultimately leads to interrupting the degradation of $\beta$-catenin by inhibition of the direct interaction between $\beta$-catenin and Herc5 in HeLa cells.

\section{DISCUSSION}

CYP1B1 is mainly known as a hydroxylase for estrogen and is widely reported to activate various types of environmental carcinogens, including aryl amines and polycyclic aromatic hydrocarbons (25). CYP1B1 shows a tumor-specific expression pattern in cells with much higher expression in tumor tissues than in normal tissues (26). Based on its function in estrogen metabolism, CYP1B1 is abundantly found in estrogen-related tissues such as mammary tissues, the uterus, and the ovary (27). However, according to Goodin et al. (28), P450 activity has cell-specific properties and HeLa cells show deficient P450 activity. Moreover, according to Nakajima et al. (29), treatment of MCF-7 cells with 
2,3,7,8-tetrachlorodibenzo-p-dioxin (TCDD) resulted in a significant increase (6 12-fold) in human CYP1A1, CYP1A2, and $C Y P 1 B 1$ mRNA levels, whereas the degree of TCDD induction for $C Y P 1 A 1$ and $C Y P 1 B 1$ was relatively weak (2 3-fold) in HeLa cells. Even though HeLa cells were derived from adenocarcinoma in the cervix, with high levels of steroids and estrogen, the basal CYP1B1 levels are very low in this cell line compared to those in other cells derived from estrogen-related tissues; this difference is attributed to the lack of P450 activity in HeLa cells (28). Therefore, the HeLa cell line is a suitable model for in vitro experiments to evaluate the effect of CYP1B1 overexpression.

DMBA is reported as a potent carcinogenic chemical belonging to polycyclic aromatic hydrocarbons (30). CYP1B1 activates DMBA through metabolic processes and CYP1B1 is critical for DMBA-induced tumor formation, which explains their reciprocally inducible relationship (31). In light of the inhibition of CYP1B1, multiple trans-stilbene analogs have been reported to suppress the CYP1 family proteins (32), and among them, TMS has been identified as a selective inhibitor of CYP1B1 (31).

In the present study, we aimed to reveal the detailed molecular mechanism of CYP1B1 in cancer progression through evaluation of the effects of CYP1B1 alteration using DMBA, TMS, and a gene overexpression/knockdown system on the signaling processes associated with carcinogenesis in HeLa cells. Based on previous findings explaining the potential carcinogenicity of CYP1B1 (6) and the correlation of Wnt/ $\beta$-catenin signaling with tumorigenesis (8), we investigated whether the $\mathrm{Wnt} / \beta$-catenin signaling pathway is responsible for CYP1B1-induced carcinogenesis. Accordingly, our data revealed that CYP1B1 protects $\beta$-catenin proteins from Herc5-mediated ISGylation which leads to the proteasomal degradation of $\beta$-catenin, and subsequently activates $\mathrm{Wnt} / \beta$ catenin signaling as validated through detection of cyclin D1 expression.

$\beta$-Catenin is a key regulator of the $\mathrm{Wnt} / \beta$-catenin signaling pathway. $\beta$-TrCP, an E3 ubiquitin ligase, executes the ubiquitination-proteasomal degradation system for cytosolic $\beta$-catenin and turns on or off $\mathrm{Wnt} / \beta$-catenin signaling via controlling the nuclear localization of $\beta$-catenin, a critical factor for target gene regulation (22). However, the ISGylation-dependent proteasomal degradation pathway was recently established as a novel mechanism of cytosolic $\beta$-catenin degradation (23). The ISGylation system, which is highly enhanced by type I IFNs (19), has been described to be orchestrated by E1 (UBE1L), E2 (UbcH8), and E3 ligase (14). Herc5 has been identified as an E3 ligase for ISGylation, which is executed by ISG15 conjugation on target proteins (18). ISG15 conjugation is known to be responsible for several biological processes other than ISGylation, including DNA repair, nuclear factor- $\kappa \mathrm{B}$ signaling, and mitotic progression (33). In the present study, we found that CYP1B1 may modulate ISGylation through regulation of ISG15.
Although CYP1B1 overexpression excessively suppressed ISG15 expression at both the mRNA and protein levels, the effects of CYP1B1 inhibition were relatively insignificant. The most reasonable explanation for these results might be the low basal expression level of ISG15 in HeLa cells. Herc5, as well as ISG15, has been reported to show low basal expression levels in most tissues (21) and is known to be constitutively promoted by type I IFNs or lipopolysaccharide (19). Accordingly, the effects of CYP1B1 suppression on Herc5 and ISG15 should be further investigated in cells under a Herc5/ISG15-abundant condition.

Taken together, the present data suggest that CYP1B1 activates $\mathrm{Wnt} / \beta$-catenin signaling through the protection and stabilization of cytosolic $\beta$-catenin levels from proteasomal degradation through suppression of ISGylation by down-regulation of ISG15 and Herc5 expression. These findings clearly show that CYP1B1 may induce carcinogenic processes in cells in accordance with $\mathrm{Wnt} / \beta$-catenin signaling activation.

\section{ACKNOWLEDGMENTS}

This research was supported by National Research Foundation of Korea (NRF) grants funded by the Korean government (MSIP) (NRF-2015R1A2A2A01003865 and NRF2015R1A5A1008958).

Received June 12, 2017; Revised June 19, 2017; Accepted June 19, 2017

\section{REFERENCES}

1. Nelson, D.R., Koymans, L., Kamataki, T., Stegeman, J.J., Feyereisen, R., Waxman, D.J., Waterman, M.R., Gotoh, O., Coon, M.J., Estabrook, R.W., Gunsalus, I.C. and Nebert, D.W. (1996) P450 superfamily: update on new sequences, gene mapping, accession numbers and nomenclature. Pharmacogenetics, 6, 142.

2. Yang, X., Zhang, B., Molony, C., Chudin, E., Hao, K., Zhu, J., Gaedigk, A., Suver, C., Zhong, H., Leeder, J.S., Guengerich, F.P., Strom, S.C., Schuetz, E., Rushmore, T.H., Ulrich, R.G., Slatter, J.G., Schadt, E.E., Kasarskis, A. and Lum, P.Y. (2010) Systematic genetic and genomic analysis of cytochrome P450 enzyme activities in human liver. Genome Res., 20, 1020-1036.

3. Shimada, T. (2017) Inhibition of carcinogen-activating cytochrome P450 enzymes by xenobiotic chemicals in relation to antimutagenicity and anticarcinogenicity. Toxicol. Res., 33, 7996.

4. Saini, S., Hirata, H., Majid, S. and Dahiya, R. (2009) Functional significance of cytochrome P450 1B1 in endometrial carcinogenesis. Cancer Res., 69, 7038-7045.

5. Delvoux, B., Groothuis, P., D'Hooghe, T., Kyama, C., Dunselman, G. and Romano, A. (2009) Increased production of $17 \beta-$ estradiol in endometriosis lesions is the result of impaired metabolism. J. Clin. Endocrinol. Metab., 94, 876-883.

6. Piotrowska, H., Kucinska, M. and Murias, M. (2013) Expres- 
sion of CYP1A1, CYP1B1 and MnSOD in a panel of human cancer cell lines. Mol. Cell. Biochem., 383, 95-102.

7. Barnett, J.A., Urbauer, D.L., Murray, G.I., Fuller, G.N. and Heimberger, A.B. (2007) Cytochrome P450 1B1 expression in glial cell tumors: an immunotherapeutic target. Clin. Cancer Res., 13, 3559-3567.

8. Akiyama, T. (2000) Wnt/ $\beta$-catenin signaling. Cytokine Growth Factor Rev., 11, 273-282.

9. Clevers, H. (2006) Wnt/ $\beta$-catenin signaling in development and disease. Cell, 127, 469-480.

10. Konigshoff, M. and Eickelberg, O. (2010) WNT signaling in lung disease: a failure or a regeneration signal? Am. J. Respir. Cell Mol. Biol., 42, 21-31.

11. Kim, W., Kim, M. and Jho, E.H. (2013) Wnt/ $\beta$-catenin signalling: from plasma membrane to nucleus. Biochem. J., 450, 921.

12. Lee, J., Li, L., Gretz, N., Gebert, J. and Dihlmann, S. (2012) Absent in Melanoma 2 (AIM2) is an important mediator of interferon-dependent and -independent HLA-DRA and HLADRB gene expression in colorectal cancers. Oncogene, 31, 1242-1253.

13. Narasimhan, J., Wang, M., Fu, Z., Klein, J.M., Haas, A.L. and Kim, J.J. (2005) Crystal structure of the interferon-induced ubiquitin-like protein ISG15. J. Biol. Chem., 280, 27356-27365.

14. Dastur, A., Beaudenon, S., Kelley, M., Krug, R.M. and Huibregtse, J.M. (2006) Herc5, an interferon-induced HECT E3 enzyme, is required for conjugation of ISG15 in human cells. J. Biol. Chem., 281, 4334-4338.

15. Shi, H.X., Yang, K., Liu, X., Liu, X.Y., Wei, B., Shan, Y.F., Zhu, L.H. and Wang, C. (2010) Positive regulation of interferon regulatory factor 3 activation by Herc5 via ISG15 modification. Mol. Cell. Biol., 30, 2424-2436.

16. Skaug, B. and Chen, Z.J. (2010) Emerging role of ISG15 in antiviral immunity. Cell, 143, 187-190.

17. Durfee, L.A., Lyon, N., Seo, K. and Huibregtse, J.M. (2010) The ISG15 conjugation system broadly targets newly synthesized proteins: implications for the antiviral function of ISG15. Mol. Cell, 38, 722-732.

18. Hochrainer, K., Mayer, H., Baranyi, U., Binder, B., Lipp, J. and Kroismayr, R. (2005) The human HERC family of ubiquitin ligases: novel members, genomic organization, expression profiling, and evolutionary aspects. Genomics, 85, 153-164.

19. Cruz, C., Ventura, F., Bartrons, R. and Rosa, J.L. (2001) HERC3 binding to and regulation by ubiquitin. FEBS Lett., 488, 74-80.

20. Wong, J.J., Pung, Y.F., Sze, N.S. and Chin, K.C. (2006) HERC5 is an IFN-induced HECT-type E3 protein ligase that mediates type I IFN-induced ISGylation of protein targets. Proc. Natl. Acad. Sci. U.S.A., 103, 10735-10740.

21. Kroismayr, R., Baranyi, U., Stehlik, C., Dorfleutner, A., Binder, B.R. and Lipp, J. (2004) HERC5, a HECT E3 ubiquitin ligase tightly regulated in LPS activated endothelial cells. J. Cell Sci.,
117, 4749-4756.

22. Liu, C., Kato, Y., Zhang, Z., Do, V.M., Yankner, B.A. and He, X. (1999) $\beta$-Trcp couples $\beta$-catenin phosphorylation-degradation and regulates Xenopus axis formation. Proc. Natl. Acad. Sci. U.S.A., 96, 6273-6278.

23. Lee, J.H., Bae, J.A., Lee, J.H., Seo, Y.W., Kho, D.H., Sun, E.G., Lee, S.E., Cho, S.H., Joo, Y.E., Ahn, K.Y., Chung, I.J. and Kim, K.K. (2010) Glycoprotein 90K, downregulated in advanced colorectal cancer tissues, interacts with CD9/CD82 and suppresses the Wnt/ $\beta$-catenin signal via ISGylation of $\beta$-catenin. Gut, 59, 907-917.

24. Gribben, J.G., Ryan, D.P., Boyajian, R., Urban, R.G., Hedley, M.L., Beach, K., Nealon, P., Matulonis, U., Campos, S., Gilligan, T.D., Richardson, P.G., Marshall, B., Neuberg, D. and Nadler, L.M. (2005) Unexpected association between induction of immunity to the universal tumor antigen CYP1B1 and response to next therapy. Clin. Cancer Res., 11, 4430-4436.

25. Shimada, T., Hayes, C.L., Yamazaki, H., Amin, S., Hecht, S.S., Guengerich, F.P. and Sutter, T.R. (1996) Activation of chemically diverse procarcinogens by human cytochrome P-450 $1 \mathrm{~B} 1$. Cancer Res., 56, 2979-2984.

26. Murray, G.I., Taylor, M.C., McFadyen, M.C., McKay, J.A., Greenlee, W.F., Burke, M.D. and Melvin, W.T. (1997) Tumorspecific expression of cytochrome P450 CYP1B1. Cancer Res., 57, 3026-3031.

27. Tsuchiya, Y., Nakajima, M., Kyo, S., Kanaya, T., Inoue, M. and Yokoi, T. (2004) Human CYP1B1 is regulated by estradiol via estrogen receptor. Cancer Res., 64, 3119-3125.

28. Goodin, M.G., Fertuck, K.C., Zacharewski, T.R. and Rosengren, R.J. (2002) Estrogen receptor-mediated actions of polyphenolic catechins in vivo and in vitro. Toxicol. Sci., 69, 354361.

29. Nakajima, M., Iwanari, M. and Yokoi, T. (2003) Effects of histone deacetylation and DNA methylation on the constitutive and TCDD-inducible expressions of the human CYP1 family in MCF-7 and HeLa cells. Toxicol. Lett., 144, 247-256.

30. Heidel, S.M., Czuprynski, C.J. and Jefcoate, C.R. (1998) Bone marrow stromal cells constitutively express high levels of cytochrome P4501B1 that metabolize 7,12-dimethylbenz[a]anthracene. Mol. Pharmacol., 54, 1000-1006.

31. Chun, Y.J. and Kim, S. (2003) Discovery of cytochrome P450 $1 \mathrm{~B} 1$ inhibitors as new promising anti-cancer agents. Med. Res. Rev., 23, 657-668.

32. Chun, Y.J., Lee, S.K. and Kim, M.Y. (2005) Modulation of human cytochrome P450 1B1 expression by 2,4,3',5'-tetramethoxystilbene. Drug Metab. Dispos., 33, 1771-1776.

33. Zou, W., Papov, V., Malakhova, O., Kim, K.I., Dao, C., Li, J. and Zhang, D.E. (2005) ISG15 modification of ubiquitin E2 Ubc13 disrupts its ability to form thioester bond with ubiquitin. Biochem. Biophys. Res. Commun., 336, 61-68. 